\title{
Pädagogische Arbeit an Schulen in sozial deprivierter Lage vor dem Hintergrund eigener biografischer Erfahrungen von Lehrkräften
}

\author{
Sabrina Rutter
}

Online publiziert: 24. Juli 2020

(C) Der/die Autor(en) 2020

Zusammenfassung Der Beitrag widmet sich der Bedeutung der sozialen Herkunft von Lehrkräften bei der Reproduktion sozialer Ungleichheit, die sich in Schulen in sozial deprivierter Lage zuspitzt. Hierzu wird der Frage nachgegangen, welche handlungsleitenden Orientierungen sich bezüglich der Unterstützung sozial benachteiligter SchülerInnen rekonstruieren lassen und inwiefern diese in Verbindung mit eigenen biografischen Erfahrungen stehen. Die Analysen zeigen, dass die pädagogische Arbeit der Lehrkräfte stark mit herkunftsspezifischen Logiken zusammenhängt. Hieraus ergeben sich wichtige Linien der zukünftigen Debatte über Bildungsungleichheit, vor allem in professionstheoretischer Hinsicht.

Schlüsselwörter Schulen in sozial deprivierter Lage $\cdot$ Reproduktion sozialer Ungleichheit · Soziale Herkunft der Lehrkräfte

\section{Educational work in schools in disadvantaged communities against the backdrop of teachers' own biographical experiences}

\begin{abstract}
The article is devoted to the importance of the social origin of teachers in the reproduction of social inequality, which is exacerbated in schools in disadvantaged communities. For this purpose, the question is investigated, which orientations can be reconstructed with regard to the support of socially disadvantaged pupils and to what extent these are connected with their own biographical experiences. The analyzes show that the pedagogical work of the teachers is strongly related to origin-specific logic. The article thus opens up important lines of the future debate on educational inequality, especially in terms of professional theory.
\end{abstract}

Dr. S. Rutter $(\bowtie)$

AG Bildungsforschung, Institut für Erziehungswissenschaft, Fakultät für Bildungswissenschaften,

Universität Duisburg-Essen, Universitätsstr. 2, 45141 Essen, Deutschland

E-Mail: sabrina.rutter@uni-due.de 
Keywords Schools in disadvantaged communities $\cdot$ Reproduction of social inequality $\cdot$ Social origin of teachers

Vor dem Hintergrund, dass die pädagogische Arbeit an Schulen in sozial deprivierter Lage unter besonders herausfordernden Bedingungen stattfindet und die SchülerInnen systematisch schlechtere Bildungschancen haben, stehen solche Schulen zunehmend im Fokus der Öffentlichkeit, Bildungspolitik und -forschung (Bremm et al. 2016). Ditton (2013) weist darauf hin, dass der Zusammenhang von sozialstrukturellen Voraussetzungen und Schulerfolg jedoch nicht kausal ist, sondern ,,vielmehr [...] zu klären [ist], inwiefern und auf welche Weise ein Kontext individuelle Haltungen, Einstellungen und Verhalten beeinflusst“ (ebd., S. 201). In dem erziehungswissenschaftlichen und bildungssoziologischen Diskurs gilt die Aufmerksamkeit dabei zumeist den Familien. Weniger beachtet wird, welche Rolle die Lehrkräfte bei der Reproduktion sozialer Ungleichheit spielen. Ausgehend von Bourdieus Passungstheorem (Bourdieu und Passeron 1971) rücken im vorliegenden Beitrag die Wechselwirkungen zwischen den sozialräumlichen Gegebenheiten der Schule und der Praxis der Lehrkräfte in den Blick. Der Fokus liegt auf den bislang eher randständig untersuchten Herkunftsmerkmalen der Lehrkräfte. Zu diesem Zweck werden Ergebnisse einer empirischen Studie zur lehrkraftseitigen Wahrnehmung von und zum pädagogischen Umgang mit sozial benachteiligten SchülerInnen vorgestellt.

Im Folgenden wird zunächst das diesem Beitrag zugrundeliegende Begriffsverständnis von Schulen in sozial deprivierter Lage erläutert (1). Daran anschließend wird unter Rückgriff auf an Bourdieu orientierte theoretische und empirische Arbeiten die Bedeutung der sozialen Herkunft der Lehrkräfte im Ungleichheitszusammenhang dargestellt (2). Nachdem in einem weiteren Schritt die forschungsleitenden Fragestellungen und die methodische Vorgehensweise der Untersuchung knapp erläutert werden (3), werden die rekonstruierten handlungsleitenden Orientierungen der Lehrkräfte präsentiert (4). Abschließend werden die Befunde zusammengefasst und aus professionstheoretischer Perspektive reflektiert (5).

\section{Begriffsverständnis von Schulen in sozial deprivierter Lage}

Mit Schulen in sozial deprivierter Lage sind zumeist Schulen in städtischen Regionen gemeint, ,in denen sich das Phänomen der wohnräumlichen Segregation besonders deutlich zeigt und für dessen Bewohnerinnen und Bewohner [diese Segregation zudem, Anm. d. Verf.] Prozesse der Exklusion und der verringerten Teilhabe mit sich zu bringen droht" (Fölker et al. 2015, S. 9). Sozialräumliche Segregation bezeichnet unterdies eine Ungleichverteilung von verschiedenen Personengruppen im Stadtgebiet, vor allem entlang sozioökonomischer sowie natio-ethno-kultureller Aspekte. Sie entsteht aufgrund von freiwilligen und unfreiwilligen Entmischungsund Polarisierungsprozessen, die unmittelbar miteinander verbunden sind (Häussermann 2008). Dort lokalisierte Schulen werden vermehrt von SchülerInnen aus sozial benachteiligten Lebensverhältnissen besucht (Fölker et al. 2015). Forschung zu Kompositionseffekten kann ferner zeigen, dass die mit dem lokalen Kontext ein- 
hergehende Zusammensetzung der SchülerInnenschaft mit schulischen Leistungen und Abschlüssen korreliert (Baumert et al. 2006).

Angesichts dieser spezifischen Voraussetzungen sind Schulen in sozial deprivierter Lage mit besonderen Herausforderungen konfrontiert, die den Schulen höhere Anstrengungen hinsichtlich der Herstellung von Bildungsgerechtigkeit abverlangen (Bremm et al. 2017). Hinzu kommt, dass diese Schulen häufig als ,Brennpunktschulen` o. Ä. bezeichnet werden (Bremm et al. 2016). Damit einhergehende Stigmatisierungsprozesse führen bspw. dazu, dass die Schulen eine vergleichsweise hohe Fluktuation unter Lehrkräften aufweisen. Gleichwohl zeigen Befunde aus der Schuleffektivitäts- und Schulentwicklungsforschung, dass es einigen Schulen trotz ungünstigeren Vorbedingungen gelingt, Lernprozesse der SchülerInnen positiv zu beeinflussen und bestmögliche Leistungsergebnisse $\mathrm{zu}$ erreichen (vgl. hierzu ausführlich Klein 2017).

In Anlehnung an Hummrich (2015) wird in diesem Beitrag Schule ,als Sozialraum im Sozialraum“ (ebd., S. 171, Hervorhebung im Original, Anm. d. Verf.) gedacht. Damit ist gemeint, dass Schule einerseits in einen Sozialraum eingebettet ist und sich entsprechend dazu positioniert und andererseits sich wahrnehmbar als Sozialraum konstituiert und deshalb auch Positionierungsleistungen vornimmt. Nach diesem Raumverständnis gestalten Schulen in sozial deprivierter Lage den Sozialraum mit und können als Ort des Sichtbarwerdens, der Reproduktion und des Abbaus sozialer Ungleichheit verstanden werden (Fölker et al. 2015). Der Themenkomplex Schule und Sozialraum umfasst schließlich mehrere Ebenen (z. B. der Gesellschaft, der Institution und des Milieus), die voneinander getrennt, aber auch in ihrem Zusammenwirken betrachtet werden müssen (Hummrich 2015). In diesem Beitrag steht die Strukturierung der pädagogischen Arbeit in und durch den Sozialraum im Mittelpunkt, vor deren Hintergrund Ermöglichung oder Verhinderung von Teilhabe entsteht.

\section{Schulischer Reproduktionsmodus sozialer Ungleichheit aus der Perspektive Bourdieus}

Die vorangegangenen Ausführungen weisen darauf hin, dass die Lehrkräfte an Schulen in sozial deprivierter Lage außerordentlich gefordert sind, die SchülerInnen in ihrer Leistungs- und Persönlichkeitsentwicklung zu unterstützen und sich mit Mechanismen der innerschulischen Herstellung bzw. Aufrechterhaltung sozialer Ungleichheit auseinanderzusetzen (Bremm et al. 2016). Bourdieus Überlegungen zur Passung des Habitus und daran anschließende Forschung ermöglichen es, Mikroprozesse der Reproduktion sozialer Ungleichheit innerhalb von Schule zu beschreiben und auf die sozialräumliche Lage zu beziehen. So basiert die Ungleichbehandlung der SchülerInnen aus Sicht von Bourdieu auf inkorporierten Wahrnehmungs-, Denk- und Handlungsschemata, die an spezifische soziale Verhältnisse gebunden sind (Bourdieu 1987). Lehrkräfte anerkennen und fördern die SchülerInnen, deren familial geprägte Einstellungs- und Verhaltensmuster zu den impliziten Strukturen und Anforderungen der Schule bzw. zu den eigenen habituellen Dispositionen passen (Bourdieu und Passeron 1971). Unter dem Stichwort „Mittelschichtsbias“ 
(Grundmann et al. 2007, S. 50) wird ausgeführt, dass sowohl die Auswahl der Bildungsinhalte als auch die Vermittlungsweise den Normen- und Wissensbeständen der bürgerlichen Mitte entsprechen.

Bourdieus Passungstheorem spielt in der Debatte über die Entstehung und Verstärkung von Bildungsungleichheit eine vergleichsweise untergeordnete Rolle; entsprechend groß sind die Forschungslücken (Betz 2015). Hervorzuheben sind die empirischen Analysen von Grundmann und seinem Forschungsteam, die lebensweltliche Bildungsprozesse in der Familie und unter Gleichaltrigen sowie die daraus resultierenden Anschlussmöglichkeiten in der Schule in den Blick nehmen und hierbei ,grundlegende Unterschiede in den Passungschancen bzw. Konfliktpotentialen" (Grundmann et al. 2003, S. 37) zwischen dem in der Familie erworbenen primären Habitus und dem von der Schule geforderten sekundären Habitus ermitteln. Die Arbeitsgruppe von Helsper und Kramer differenziert u.a. die Ebene der Institution weiter aus: Zum einen stellen die ForscherInnen heraus, dass es unterschiedliche milieuspezifische primäre Habitusformen gibt, die von einer explizit formulierten Bildungsdistinktion bis hin zu einer Distanz- und Fremdheitshaltung zu schulischer Bildung reichen (Kramer et al. 2009). Zum anderen gehen sie nicht von einem allgemeingültigen sekundären Habitus aus, sondern machen auf bedeutsame schulkulturelle Unterschiede aufmerksam. Anhand von Fallstudien zu Schulen unterschiedlicher Schulformen kann die Arbeitsgruppe zeigen, dass die untersuchten Einzelschulen differente sekundäre Habitus einfordern, die entlang des jeweiligen Entwurfs der idealen SchülerInnen konstruiert werden. Damit einher gehen milieuspezifische Anknüpfungs- und Abstoßungsverhältnisse (Helsper et al. 2009).

Gerade in benachteiligten Sozialräumen wiegen Fragen zu Passungsverhältnissen schwer, da Stadtteile mit segregierten BewohnerInnenschaften weniger Optionen zum Erwerb der in Schule anschlussfähigen Kompetenzen und Fähigkeiten bieten (Bremm et al. 2016). Studien, die sich explizit auf den Einfluss sozialstruktureller Kontextbedingungen auf das pädagogische Handeln konzentrieren, entstehen erst in jüngerer Zeit. Etwa können Fölker und Hertel (2015) unterschiedliche Formen der Bezugnahme der Lehrkräfte auf die SchülerInnen an zwei Großstadtschulen in sozial deprivierter Lage herausarbeiten. Die Befunde umfassen in der ersten Fallstudie sowohl migrationsspezifische Stereotype als auch eine Fokussierung auf vermeintlich problematische Verhaltensweisen bei gleichzeitiger Ausblendung sozialer Hintergründe. Mit Blick auf die zweite Fallstudie sind insbesondere Anschauungen bedeutsam, die die sozioökonomische Benachteiligung in den Mittelpunkt stellen. Darüber hinaus scheint die eher defizitorientierte Sichtweise den Umgang mit den Kindern und Jugendlichen maßgeblich auszurichten: In der ersten Fallstudie geht es vorwiegend um Praktiken der An- und Einpassung der SchülerInnen entweder bezogen auf ethnische und geschlechtsbezogene Normvorstellungen oder hinsichtlich schulisch erwarteter Verhaltens- und Umgangsformen. In der zweiten Fallstudie bildet eine engmaschige Betreuung der SchülerInnen und Eltern den Schwerpunkt der pädagogischen Arbeit. Racherbäumer (2017) analysiert Einstellungen und Handlungsstrategien von Lehrkräften an Schulen in sozial deprivierter Lage mit dem Fokus auf LehrerInnen-SchülerInnen-Beziehungen. Es zeigen sich verschiedene Muster, die sich bezüglich der Ausgestaltung und antizipierten Wirkung unterscheiden. Insgesamt verweisen die exemplarisch zitierten Arbeiten von Fölker et al. (2015) 
und Racherbäumer (2017) zwar auf vielfältige Bemühungen der Lehrkräfte, der sozialen Benachteiligung der SchülerInnen entgegenzuwirken. Allerdings wird auch deutlich, dass die Lehrkräfte den Eltern bisweilen mangelnde Bildungs- und Erziehungsfähigkeit attestieren und diese als ursächlich für Passungskrisen ausweisen.

Auch wenn die vorausgehenden Studien einen Einblick geben, wie es zu Diskrepanzen zwischen dem in der Familie erworbenen Habitus und den Ansprüchen der Schule kommen kann, besteht weiterhin ein Forschungsdesiderat in der systematischen Exploration des Zusammenhangs von Hintergrundmerkmalen von Lehrkräften und ihren beruflichen Orientierungen und Praktiken. Neuere Untersuchungen der Arbeitsgemeinschaft Bremer, Lange-Vester und Teiwes-Kügler, die sich mit den Habitusformen und der Milieuzugehörigkeit der am Schulgeschehen Beteiligten auseinandersetzen, können erste Hinweise darauf geben, dass die habituellen Dispositionen von Lehrkräften an die jeweilige soziale Lage bzw. das soziale Milieu gebunden sind und an die SchülerInnen herangetragen werden. Die Studien zeigen ferner, wie auch andere Arbeiten zum LehrerInnenhabitus (u.a. Kramer und Pallesen 2018; Helsper 2018), dass die Habitus- und Milieuformen der Lehrkräfte zwar vielfältig sind, aber die Heterogenität der Lehrkräfte eingeschränkter ist als die der SchülerInnen (u. a. Bremer und Lange-Vester 2014; Lange-Vester und TeiwesKügler 2014).

\section{Forschungsdesign}

Vor diesem Hintergrund betrachtet der vorliegende Beitrag die Erfahrungen von Grundschullehrkräften an Schulen in sozial deprivierter Lage und setzt diese in Beziehung zu ihrer eigenen sozialen Herkunft. Konkret wird danach gefragt, welche handlungsleitenden Orientierungen sich hinsichtlich der Unterstützung sozial benachteiligter SchülerInnen rekonstruieren lassen und inwiefern diese in Verbindung mit eigenen biografischen Erfahrungen stehen. Darüber hinaus ist von Interesse, wie die Lehrkräfte die spezifische Situation ihrer Schule deuten und welche Auswirkungen die sozialstrukturellen Kontextbedingungen der Schule auf die Wahrnehmung von und den Umgang mit einer segregierten SchülerInnenschaft haben.

Empirische Grundlage dieses Beitrags bilden zwölf themenzentrierte Interviews sowie sechs Gruppendiskussionen mit Grundschullehrkräften, die im Rahmen des Forschungsprojektes ,Soziale Kompetenzen von Lehrkräften zur Entwicklung von Bildungschancen für Kinder in besonderen Lebenslagen" ${ }^{11}$ gewonnen und für ein bereits erfolgreich abgeschlossenes Promotionsvorhaben vertiefend ausgewertet wurden. In diesem Beitrag werden ausschließlich die Lehrkräfte berücksichtigt, die an städtischen Grundschulen in sozial deprivierter Lage tätig sind ${ }^{2}$.

Ausgewertet wurden die Interviews und Gruppendiskussionen mit der dokumentarischen Methode nach Bohnsack (1989), die darauf zielt, implizite und inkorporier-

\footnotetext{
${ }^{1}$ Das Projekt wurde in der Zeit von 2014-2017 mit Mitteln des Ministeriums für Innovation, Wissenschaft und Forschung des Landes Nordrhein-Westfalen an der Universität Duisburg-Essen gefördert.

${ }^{2}$ Im Rahmen des Projekts wurden Schulen mit unterschiedlichen sozialstrukturellen Kontextbedingungen einbezogen.
} 
te Wissensbestände der Erforschten in ihren spezifischen Erlebniszusammenhängen zu rekonstruieren. Dieses atheoretische Wissen ist den Personen in der Regel nicht reflexiv zugänglich (Bohnsack und Pfaff 2010). Unter Rückgriff auf die Wissenssoziologie von Mannheim (1964) und das Habitus-Konzept von Bourdieu (1987) wird davon ausgegangen, dass es sich bei dem atheoretischen Wissen um kollektiv geteilte Erfahrungen handelt, die in konjunktiven Erfahrungsräumen herausgebildet werden und die die Handlungspraxis strukturieren. Dabei verbinden konjunktive Erfahrungen Personen auch jenseits persönlicher Bekanntschaft und direkter Interaktion miteinander, vielmehr geht es um strukturidentische Erfahrungen aufgrund einer gemeinsamen Sozialisationsgeschichte bzw. gleichartiger sozialer Lebensumstände. Demnach kann in einem konjunktiven Erfahrungsraum von einer habituellen Übereinstimmung der sozialen Akteure ausgegangen werden (Bohnsack 2014).

Im Rahmen der Dissertation wurde über die Rekonstruktion der Orientierungsrahmen der einzelnen Fälle hinaus eine sinngenetischen Interpretation (Vergleich der Fälle hinsichtlich inhaltlicher Gemeinsamkeiten und Unterschiede) sowie eine soziogenetischen Interpretation (Analyse der jeweiligen sozialen Zusammenhänge und Genese handlungsleitender Orientierungen) vorgenommen (Bohnsack und Pfaff 2010). In diesem Beitrag kann das Auswertungsverfahren nicht in der Breite dargestellt werden; im Vordergrund steht die sinngenetische Typenbildung.

\section{Ergebnisse}

Durch das rekonstruktive Verfahren der dokumentarischen Methode konnten zwei Muster der lehrkraftseitigen Unterstützung herausgearbeitet und fallübergreifend abstrahiert werden, die sich als wesentlich für die Konstruktion der SchülerInnenund Elternschaft und das berufliche Selbstkonzept darstellten: eine aus scheinbar homologen biografischen Erfahrungen resultierende Einsatzbereitschaft sowie ein anerzogenes, prinzipiengeleitetes Engagement. Am Beispiel von zwei Lehrkräften sollen im Folgenden die grundlegenden handlungsleitenden Orientierungen präsentiert werden.

\subsection{Engagement im Kontext scheinbar homologer biografischer Erfahrungen}

Kennzeichnend für diesen Typus ist, dass das Einzugsgebiet der Schule als ein Ort der sozialen Benachteiligung stilisiert wird und die Lehrkräfte eine auf mehreren Ebenen stark hilfsbedürftige SchülerInnenschaft entwerfen:

Also ich würde sagen, dass speziell für unsere Schule, die ich jetzt mal als Brennpunktschule bezeichne, viele Kinder aus bildungsfernen Elternhäusern, sozial schwachen Familien in allen möglichen Lebensbereichen und auch in der Schule, einen sehr großen Bedarf haben an zusätzlicher Hilfe. Ich würde sagen, wir bräuchten viel mehr Personal, gerade was Sozialarbeiter angeht, müsste eigentlich jeder Jahrgang einen eigenen haben. Weil die Bedürfnisse so groß sind und die Unterstützung einfach durch Außenstehende- dass ich sagen würde, dort bräuchte man viel mehr- auch viel mehr Förderlehrkräfte speziell 
für unsere Klientel. (Interview Frau Kamper, Z. 141-152)

An dem Interviewausschnitt wird ersichtlich, dass die sozial deprivierte Lage der Schule einen zentralen Faktor in der Perspektive von Frau Kamper einnimmt. So charakterisiert sie ihre Schule als „Brennpunktschule“ und verweist unmittelbar auf den hohen Anteil von SchülerInnen aus „,bildungsfernen Elternhäusern“ und ,sozial schwachen Familien“. Die Beschreibung, ,in allen möglichen Lebensbereichen“ drückt hierbei die Vielfalt an individuellen Problemlagen aus. Aufgrund des damit in Verbindung stehenden ,sehr großen Bedarf[s] an zusätzlicher Hilfe“ bräuchte die Schule aus Sicht von Frau Kamper mehr Rückhalt speziell in den Bereichen Schulsozialarbeit und sonderpädagogische Förderung. An anderer Stelle führt Frau Kamper den enormen Unterstützungsbedarf aus:

Das bezieht sich auf jeden Fall auf die materielle Ausstattung der Kinder. Dass ich auch oft losgegangen bin und Sachen gekauft habe, Hefte besorgt habe. [...] Dass ich mit den Eltern über Sachen wie Körperpflege gesprochen habe und denen erklärt habe, ihr Kind muss warm angezogen sein, das muss sich morgens die Zähne putzen, die Haare kämmen. (Interview Frau Kamper, Z. 957-983)

Aufgrund der Wahrnehmung der spezifischen Bedarfe orientiert Frau Kamper ihre Praxis vor allem am Ausgleich materieller Bedürftigkeit der Kinder sowie an alltagspraktischer Beratung der Eltern. Implizit erscheint, dass die SchülerInnen nicht nur keine schulbildungsbezogene Förderung durch die Eltern erfahren, sondern überdies Regeln und Strukturen in der Familie fehlen. Lehrkräfte müssen demnach permanent die wahrgenommenen mangelnden erzieherischen Fähigkeiten der Eltern kompensieren. Ein wesentliches Merkmal dieses Musters ist dabei, dass das fachliche Lernen in den Hintergrund tritt: Die Lehrkräfte thematisieren über die gesamten Interviews hinweg weder die schulische Kompetenzvermittlung noch die Herstellung von Lernmotivation. Im Fokus steht der durch die schwierigen Lebensverhältnisse induzierte Bedarf an Erziehung und Kompensation. Der Bezug auf die SchülerInnen und Eltern bewegt sich derweil nah am Modus einer sozialpädagogischen Einflussnahme.

Obschon die als prekär wahrgenommenen familialen Verhältnisse der SchülerInnen in der Perspektive von Frau Kamper die Lernvoraussetzungen beeinträchtigen und eine ständige Herausforderung für die pädagogische Arbeit darstellen, scheinen die genannten Defizite schulisch handhabbar bzw. bearbeitbar zu sein. In diesem Zusammenhang weisen die Lehrkräfte dieses Typus explizit auf einen wahrgenommenen ähnlichen biografischen Erfahrungshintergrund wie den der SchülerInnen hin:

Ich würde schon sagen, dass ich Parallelen sehe aus meinem Leben zum Leben der Kinder. Ich denke, das ist auch ein Grund, warum ich gerade zu Beginn meines Berufs mir eine Schule ausgesucht habe, in der gerade sozial schwache Kinder mit vielen Problemen sind. Und da würde ich auch einfach sagen, ich komme halt auch aus einer eher bildungsfernen Familie. [...] Dass ich jetzt rückblickend auch beurteilen kann, dass wir auch nicht besonders viel Geld hatten, wir auch in einer kleinen Wohnung gewohnt haben. (Interview Frau Kamper, Z. 1289-1301) 
Die angenommenen lebensgeschichtlichen „Parallelen“ sind aus Sicht von Frau Kamper ein Grund dafür, dass sie sich zu Berufsbeginn eine Schule mit „,sozial schwache[n] Kinder[n] mit vielen Problemen“ ausgesucht hat. Hierbei betont Frau Kamper die gezielte Entscheidung für eine Schule mit einer stark hilfsbedürftigen SchülerInnenschaft. Gemeinsamkeiten zwischen ihr und den SchülerInnen bestehen der Lehrerin zufolge hinsichtlich der Aufwachsensbedingungen: So verortet Frau Kamper sich selbst in einer ,eher bildungsfernen Familie“ und begründet dies u.a. mit finanzieller Knappheit und bescheidenen Wohnverhältnissen.

Die eigenen biografischen Erfahrungen sind darüber hinaus konstitutiv für die pädagogische Arbeit; aus ihnen leiten die Lehrkräfte dieses Musters spezifische Fähigkeiten für den Umgang mit den SchülerInnen und Eltern ab:

Ich denke, dass ich mich besonders in solche Familien einfühlen kann, die Probleme haben, die sich nicht so gut kümmern können. Dass ich besonders empathisch bin, so ein Gespür habe für den Umgang mit diesen Kindern und Eltern. Dass ich nachvollziehen kann, dass Kinder nicht so einen geraden Weg gehen, sondern dass halt bestimmte Lebensumstände dazu führen, dass Kinder halt Auffälligkeiten haben oder dass die- ja wie soll ich das sagen? Dass die Schullaufbahn nicht gerade verläuft, sondern dass es halt mal Einbrüche gibt. So wie es bei mir halt auch gewesen ist. Ich hatte häufige Schulwechsel, hatte dann wirklich Leistungshochs und dann kamen wieder extreme Tiefs, aus denen ich mich hochgezogen habe. (Interview Frau Kamper, Z. 1397-1410)

Im Zentrum des Interviewausschnitts steht die Vorstellung eines besonderen $\mathrm{Zu}$ gangs zu Familien, ,die Probleme haben, die sich nicht so gut kümmern können“. Bezeichnend für diesen Typ ist, dass sich die Lehrkräfte aufgrund unterstellter homologer Erfahrungen mehr Sensibilität und Empathie für den Umgang mit SchülerInnen und Eltern zuschreiben, die sie als ähnlich zu sich selbst zu erkennen glauben. So führt nach Einschätzung von Frau Kamper der eigene schulische Werdegang, der durch „häufige Schulwechsel“ sowie „Leistungshochs“ und „extreme Tiefs“ gekennzeichnet ist, zu mehr Verständnis dafür, dass Kinder aus schwierigen Lebensverhältnissen nicht immer einen ,geraden Weg“ gehen, sondern aufgrund ,bestimmter Lebensumstände“ „Auffälligkeiten“ zeigen und die Schullaufbahn „Einbrüche“ aufweist.

Ein weiteres wesentliches Merkmal dieses Musters ist die wahrgenommene exklusive Beziehung zu SchülerInnen und Eltern mit einem vermeintlich konjunktiven Erfahrungshintergrund:

Dass die Kinder auch sehr offen zu mir sind, ist eine Stärke. Also wirklich mit ihren Sorgen zu mir kommen, das auch ansprechen können, egal ob es jetzt den Raum Schule oder das Zuhause betrifft. Also das haben auch schon andere Lehrer gesagt, dass die Kinder wirklich mit vielen Dingen zu mir kommen und mir anvertrauen, was die zum Beispiel aus ihrer Klasse nicht kennen. Aber nicht nur die Kinder, auch die Eltern auf mich zukommen und mich um Rat fragen. (Interview Frau Kamper, Z. 568-582)

Frau Kamper übernimmt aus ihrer Sicht sowohl für die SchülerInnen als auch für die Eltern die Rolle einer Vertrauensperson. Als positiv hebt sie die Offenheit der 
Kinder im Hinblick auf die Äußerung von schulischen und familialen Sorgen hervor. Auffallend ist bei diesem Typ die Abgrenzung von den KollegInnen: Frau Kamper betont die Rückmeldung anderer Lehrkräfte, dass das vertrauensvolle Verhältnis zwischen ihr und den SchülerInnen außergewöhnlich sei. Diese Äußerung kann auf die individuelle Position dieses Typus innerhalb der Schule bzw. des Kollegiums verweisen: Frau Kamper scheint mit ihrer Orientierung in gewisser Distanz zu der dominanten Kultur der Einzelschule und zu dem üblichen Spektrum der Lehrkräfte zu stehen.

Tendenziell erweisen sich die vorgeblich biografisch erworbenen Handlungskompetenzen in der Selbstdarstellung der Lehrkräfte passgenau zu den wahrgenommenen einzelschulspezifischen Herausforderungen. So betonen die Lehrkräfte ihre spezielle Befähigung, mit der für die jeweilige Schule anscheinend charakteristischen SchülerInnen- und Elternschaft bewusster und erfolgreicher umgehen zu können. Der eigene biografische Erfahrungshintergrund wird als wichtige Ressource für die pädagogische Arbeit und als entscheidendes Differenzmerkmal im Kollegium verstanden. Vor dem Hintergrund, dass Frau Kamper durch ihre scheinbar eher benachteiligten Herkunftsverhältnisse vermutlich zu einer unterrepräsentierten sozialen Gruppe in der LehrerInnenschaft zählt, kann die ausgewiesene Nähe zu deprivierten Soziallagen eine (unbewusste) Strategie darstellen, um ihre möglicherweise randständige Stellung in der Einzelschule zu verbessern.

\subsection{Anerzogenes, prinzipiengeleitetes Engagement}

Im Gegensatz zum ersten Muster wird beim zweiten jetzt darzustellenden Typ das lokale Umfeld der Schule und damit einhergehend die soziale Zusammensetzung der SchülerInnenschaft nicht generell problematisiert:

Es ist bunt gemischt. Also wir haben tatsächlich Kinder von den Ärzten oben aus dem Krankenhaus, eine Bankerin habe ich als Mutter und so weiter. Ich habe aber auch Hartz IV-Empfänger-Eltern. Also wirklich bunt gemischt. Ja und wir gelten laut den Zahlen sogar teilweise als Problemschule. Wir haben Kinder mit Migrationshintergrund von fünfundzwanzig bis zweiunddreißig Prozent, je nachdem hat unser Chef mal gesagt. Empfinden wir aber noch nicht so. (Interview Herr Jansen, Z. 657-682)

Herrn Jansen zufolge sind die SchülerInnen mit Blick auf den sozioökonomischen Hintergrund sehr heterogen. So unterrichtet er sowohl Kinder, deren Eltern gesellschaftlich angesehene Berufe ausüben und ein tendenziell überdurchschnittliches Einkommen erzielen, als auch Kinder, deren Eltern arbeitslos sind und staatliche Transferleistungen beziehen. Zudem weisen über ein Viertel der SchülerInnen einen Migrationshintergrund auf. Infolge dieses hohen Anteils gelte die Schule ,sogar teilweise als Problemschule“. Eine durch Migration gekennzeichnete SchülerInnenschaft wird von Herrn Jansen (und dem Kollegium) jedoch nicht per se als Problem wahrgenommen. Die Ablehnung des pauschal negativ konnotierten Begriffs „Problemschule" steht hierbei im Kontrast zum ersten Typus, bei dem die Lehrkräfte ihre Schule mitunter selbst als „Brennpunktschule“ bezeichnen. Die vorherigen Rekonstruktionen konnten zeigen, dass die negative Etikettierung der Schule eine spezielle 
Funktion erfüllt: Die Lehrkräfte messen ihrer herkunftsbedingten Perspektive auf die SchülerInnen- und Elternschaft besondere Relevanz für die pädagogische Arbeit bei und entwerfen darauf aufbauend ein spezifisches Kompetenzprofil, das den wahrgenommenen Bedarfen ihrer Schule entspricht.

Der entscheidende Unterschied zum erstgenannten Typ ist, dass die Fokussierung auf und das Engagement für bestimmte SchülerInnen nicht mit der Annahme gemeinsam geteilter biografischer Erfahrungen in Verbindung steht, sondern um qua in der eigenen Erziehung aufgenommener Grundsätze:

Also natürlich ging es mir sehr gut. Meine Kindheit und mein- also wenn ich
jetzt die Kinder sehe, die ich unterrichte, dann ging es mir sehr sehr gut. [...] Ich
bin einfach froh, selbst eine gute Erziehung genossen zu haben und manchmal
versuche ich mich auch daran zu erinnern. Auch wenn ich persönlich vielleicht
auch mal ein bisschen Scheiße gemacht habe. Am Ende muss ich ja trotz-
dem [...] eine gute Erziehung genossen haben, worauf ich jetzt zurückgreife.
Auch wenn ich damals zwischendurch vom rechten Weg vielleicht ein bisschen
abgekommen bin, aber ich kann ja trotzdem mich dieser Dinge immer noch be-
dienen und die auch irgendwie abrufen. (Interview Herr Jansen, Z. 2629-2672)

Der Interviewausschnitt zeigt, dass die Ressourcen der eigenen Herkunft ihren Ursprung in grundlegenden Wertvorstellungen haben, die in der Erziehung vermittelt wurden. So schildert Herr Jansen, dass es ihm im Vergleich zu den SchülerInnen, die er jetzt unterrichtet, in seiner eigenen Kindheit ,sehr sehr gut“ ging. Denn obwohl er ,vielleicht auch mal ein bisschen Scheiße gemacht“ hat und er ,damals zwischendurch vom rechten Weg vielleicht ein bisschen abgekommen“ ist, hat er „eine gute Erziehung genossen“ und kann sich dieser ,immer noch bedienen und die auch irgendwie abrufen“. Herr Jansen deutet somit zwar eine gewisse Verbundenheit mit seinen SchülerInnen an, konstruiert dabei aber im Gegensatz zu Frau Kamper keinen konjunktiven Erfahrungsraum.

Charakteristisch für dieses Muster ist die praktische Adaption der in der Erziehung aufgenommenen Prinzipien für das eigene pädagogische Handeln. So geht es den Lehrkräften vornehmlich darum, möglichst alle SchülerInnen optimal zu fördern. In diesem Sinne steht bei Herrn Jansen die Unterstützung von verhaltensauffälligen Kindern im Vordergrund des Interviews. Am Beispiel eines Schülers mit ADHS illustriert er, dass der Status ,Problemkind“ keine Eigenschaft, sondern vielmehr ein zeitlich begrenzter Zustand bzw. eine Lebensphase darstellt und veränderbar ist:

Man kann die eigentlich bekommen. Nicht alle, je nach Schweregrad oder je nach Erscheinungsbild der Krankheit. [...] Ich habe zum Beispiel einen in der Klasse, der ist super lieb, der würde vielleicht als Problemkind gelten bei vielen Lehrern, aber für mich ist das kein Problemkind. Der hat zwar- das ist eine krasse Story, die Mutter wollte den umbringen. [...] Als der Mann sich getrennt hat, dann hat sie irgendwie einen Tötungsversuch vorgenommen und hat sich aber während des Vorgangs das- hat sie die Polizei und einen Krankenwagen gerufen, sich selbst angezeigt. [...] Der hat halt schwer ADHS, aber für mich gilt der trotzdem nicht als Problemkind, weil sein Vater super engagiert ist, das mit der Mutter sich wieder halbwegs eingerenkt hat. [...] Und dem geht es so 
ganz gut, der ist kein Problemkind mehr, das war er vielleicht mal. (Interview Herr Jansen, Z. 835-882)

Schwere Fälle von ADHS stellen Herrn Jansen zufolge an sich kein Problem dar. Er argumentiert, dass man solche SchülerInnen ,eigentlich bekommen“ kann. Diese Auffassung schränkt er durch den „Schweregrad“ oder das „Erscheinungsbild der Krankheit" ein. Die Betonung der positiven Charaktereigenschaft des Jungen kann als Opposition zu der antizipierten Sichtweise der KollegInnen gedeutet werden. Herr Jansen ergänzt, dass der Junge angesichts seiner ADHS-Erkrankung und seiner „krasse[n] Story“ „vielleicht mal ein Problemkind“ gewesen sei. Hierbei bringt er seine Eindrücke zu den kritischen Lebensereignissen des Schülers in eine (suggerierte) kausale Ordnung, die auf den Befund, schwerer Fall von ADHS ‘ hinausläuft. Der Junge ist für ihn trotz aller Umstände kein „Problemkind“, was er mit einem Prozess der Stabilisierung von Familienverhältnissen begründet. Hier bestätigt Herr Jansen, dass der temporäre Charakter und die unterschiedlichen Ursachen und Ausprägungen von Verhaltensauffälligkeiten bei ADHS für ihn gegen die verallgemeinerte Zuschreibung „Problemkind“ sprechen.

Insgesamt zeichnet sich dieser Typ durch den Anspruch von weitestgehender Unterstützung aller SchülerInnen aus. Die praktische Umsetzung umfasst die Distanzierung von einer verallgemeinernden Sichtweise auf vermeintliche ,Problemkinder “ und führt im Fall von Herrn Jansen darüber hinaus auch zu Widerstand gegen die Schulleitung und Elternschaft: So fordern Eltern im Zuge eines Konflikts mit einem anderen Kind den Schulverweis einer Schülerin. Herr Jansens tritt erfolgreich für den Verbleib des Mädchens an der Schule ein:

Mit Anna gab es viel Stress [...] und da war ich derjenige, der sich für Anna eingesetzt hat. [...] Das ging mir da so ein bisschen schnell und da wollten die Eltern die [aus der Schule] mobben. [...] Meinem Chef habe ich auch gesagt, wenn du die von der Schule schmeißen willst, dann mach das. Dann sag das aber auch so. Wir reißen uns hier seit drei Monaten den Hintern auf für dieses Kind und es kann jetzt nicht sein, dass die geht. Und dann haben wir auch in der Konferenz, er hat sich enthalten als Schulleiter, [...] die Elternvertretung bearbeitet und dann haben die das gestimmt, was wir wollten. (Interview Herr Jansen, Z. 2543-2599)

Für Herrn Jansen kommt es nicht in Frage, der Forderung des Schulverweises seitens der Elternschaft nachzugeben. Aus seinem Blickwinkel reagieren die Eltern vorschnell und unangemessen. Bei einem Gespräch mit dem Schulleiter über die Auseinandersetzungen mit den Eltern führt Herr Jansen die harte Arbeit an dem Mädchen als Argument für ihr Bleiben an. Insgesamt zeigt sich, dass Herr Jansen eine Haltung vertritt, der zufolge ein offensichtlich und auf unbestimmte Zeit schwieriges Kind und der entsprechende Einsatz der Lehrkräfte Gründe für eine gemeinsame Beschulung sind. Diese Schilderung kann auf eine hegemoniale Orientierung innerhalb der Einzelschule hindeuten, die Grundlage für sein selbstsicheres Auftreten ist. Möglichweise eröffnet seine Schule für engagierte Lehrkräfte einen entsprechenden Handlungsspielraum, oder Herr Jansen nimmt für sich aufgrund sei- 
ner offenbar eher privilegierten Herkunftsverhältnisse Gestaltungsmöglichkeiten in Anspruch.

\section{Zusammenfassung und Diskussion}

In diesem Beitrag ging es darum, die Bedeutung der sozialen Herkunft der Lehrkräfte für die pädagogische Arbeit an Schulen in sozial deprivierter Lage zu untersuchen. Mittels der dokumentarischen Methode wurden zwei Muster handlungsleitender Orientierungen hinsichtlich der Unterstützung sozial benachteiligter SchülerInnen identifiziert, die einerseits als Engagement im Kontext scheinbar homologer biographischer Erfahrungen sowie andererseits als anerzogenes, prinzipiengeleitetes Engagement zusammengefasst werden können. Bei dem ersten Typus stellt vor allem die Konstruktion eines konjunktiven Erfahrungsraums eine gewichtige Dimension in Bezug auf die Wahrnehmung der SchülerInnen- und Elternschaft sowie die damit einhergehende Deutung von familialen Problemlagen und pädagogischen Handlungsbedarfen, der Zuschreibung von praktischen Handlungswissen und spezifischen Kompetenzen, der Erfahrung von Selbstwirksamkeit sowie der Bereitschaft, Verantwortung für die Entwicklung von Bildungschancen für diese bestimmten Kinder zu übernehmen, dar. So betonen die Lehrkräfte, dass ihr besonderer Zugang zu sozial benachteiligten SchülerInnen auf impliziten, inkorporierten Wissen beruht, das aus der Erfahrung gleichartiger Aufwachsensbedingungen resultiert. Auf dieses Phänomen wurde bislang lediglich in der Forschung zu beruflichen Fremdund Selbstkonzepten von Lehrkräften mit Migrationshintergrund (u. a. Fabel-Lamla und Klomfaß 2014; Rotter 2014) verwiesen. Die vorliegenden Analysen zeigen indes, dass die angenommenen ähnlichen biografischen Erfahrungen der Lehrkräfte nicht ausschließlich im Zusammenhang von Migration zu verorten sind. Demgegenüber beruhen bei dem zweiten Typus das Problembewusstsein und die darauf abgestimmten beruflichen Praktiken gerade nicht auf einer angenommenen habituellen Übereinstimmung. Gleichwohl kommen auch bei diesem Muster die eigenen biografischen Erfahrungen in Form von universal geltenden Prinzipien bzw. grundlegenden Wertvorstellungen in der pädagogischen Arbeit zum Tragen.

Tendenziell können sich die konjunktiven Erfahrungen im Einzelfall als eine Ebene erweisen, mit der eine Brücke zu bestimmten Formen von sozialer Benachteiligung hergestellt werden kann. Aus professionstheoretischer Perspektive sind die vorliegenden Befunde jedoch kritisch einzuschätzen: Auf der einen Seite besteht die Gefahr ,blinder Flecken' für die pädagogische Arbeit, indem die eigenen biografischen Erfahrungen der Lehrkräfte unreflektiert auf die SchülerInnen und Eltern übertragen werden und in der Folge die Besonderheiten der jeweiligen Lebensumstände unberücksichtigt bleiben. Etwa ist fraglich, inwiefern die Lehrkräfte des ersten Typus tatsächlich Einsicht in die vielfältigen deprivierten Soziallagen der SchülerInnen haben. Zum einen reicht ein bestimmtes Deutungsmuster nicht aus, um in der Schule Anschlussmöglichkeiten für sozial benachteiligte SchülerInnen zu eröffnen - die Grundlegung eigener biografischer Erfahrungen würde eine Deprofessionalisierung der Lehrkräfte gleichkommen. Vielmehr geht es darum, mit umfassenden pädagogischen (Fach-)Wissen Beziehungen zu und Interaktionen mit 
SchülerInnen auszugestalten. Zum anderen würde ein besonderer Zugang zu sozial benachteiligten SchülerInnen auf Grundlage angenommener geteilter biografischer Erfahrungen bedeuten, dass es sich hierbei um Wissensbestände handelt, die nicht im Studium und Beruf erworben werden können. Gleichberechtigung und Chancengleichheit würde folglich nur durch eine adäquate Repräsentation jeglicher sozialer Gruppen bzw. Differenzmerkmale unter den Lehrkräften hergestellt werden können (vgl. hierzu auch Fabel-Lamla und Klomfaß 2014). Weiterhin weisen die Analyseergebnisse auf eine fehlende Sensibilität und Offenheit der Lehrkräfte gegenüber der Verschiedenheit der SchülerInnen hin. Lehrkräfte des ersten Typus beziehen sich maßgeblich auf die sozialbedingten Problemlagen, Lehrkräfte des zweiten Typus auf Verhaltensauffälligkeiten. Weitere Aspekte, die zu unterschiedlichen Lernvoraussetzungen und Entwicklungsbedingungen in der Schule sowie zu einem erhöhten Risiko für eine schulische Ungleichbehandlung führen können, wie bspw. bildungssprachliche Kompetenzen werden in den Interviews kaum thematisiert.

Aus diesen Gründen erscheint die permanente Reflexion und Kontrolle des Anteils der eigenen sozialen Herkunft von Lehrkräften bei der Prozessierung von Bildungsungleichheit unabdingbar, um mögliche Passungsprobleme rechtzeitig durch geeignete Handlungsstrategien abzumildern bzw. zu verhindern.

Funding Open Access funding provided by Projekt DEAL.

Open Access Dieser Artikel wird unter der Creative Commons Namensnennung 4.0 International Lizenz veröffentlicht, welche die Nutzung, Vervielfältigung, Bearbeitung, Verbreitung und Wiedergabe in jeglichem Medium und Format erlaubt, sofern Sie den/die ursprünglichen Autor(en) und die Quelle ordnungsgemäß nennen, einen Link zur Creative Commons Lizenz beifügen und angeben, ob Änderungen vorgenommen wurden.

Die in diesem Artikel enthaltenen Bilder und sonstiges Drittmaterial unterliegen ebenfalls der genannten Creative Commons Lizenz, sofern sich aus der Abbildungslegende nichts anderes ergibt. Sofern das betreffende Material nicht unter der genannten Creative Commons Lizenz steht und die betreffende Handlung nicht nach gesetzlichen Vorschriften erlaubt ist, ist für die oben aufgeführten Weiterverwendungen des Materials die Einwilligung des jeweiligen Rechteinhabers einzuholen.

Weitere Details zur Lizenz entnehmen Sie bitte der Lizenzinformation auf http://creativecommons.org/ licenses/by/4.0/deed.de.

\section{Literatur}

Baumert, J., Stanat, P., \& Watermann, R. (2006). Schulstruktur und die Entstehung differenzieller Lernund Entwicklungsmilieus. In J. Baumert, P. Stanat \& R. Watermann (Hrsg.), Herkunftsbedingte Disparitäten im Bildungswesen. Vertiefende Analysen im Rahmen von PISA 2000 (S. 95-188). Wiesbaden: Springer VS.

Betz, T. (2015). Ungleichheitsbezogene Bildungsforschung - Lehrkräfte im Fokus. Zeitschrift für Soziologie der Erziehung und Sozialisation (ZSE), 2015(4), 339-343.

Bohnsack, R. (1989). Generation, Milieu und Geschlecht. Ergebnisse aus Gruppendiskussionen mit Jugendlichen. Wiesbaden: Springer VS.

Bohnsack, R. (2014). Rekonstruktive Sozialforschung: Einführung in qualitative Methoden (9. Aufl.). Opladen: UTB.

Bohnsack, R., \& Pfaff, N. (2010). Die dokumentarische Methode: Interpretation von Gruppendiskussionen und Interviews. Enzyklopädie Erziehungswissenschaft online. (S. 1-55).

Bourdieu, P. (1987). Sozialer Sinn. Kritik der theoretischen Vernunft. Frankfurt a. M.: Suhrkamp. 
Bourdieu, P., \& Passeron, J.-C. (1971). Die Illusion der Chancengleichheit. Untersuchungen zur Soziologie des Bildungswesens am Beispiel Frankreichs. Stuttgart: Klett.

Bremer, H., \& Lange-Vester, A. (2014). Die Pluralität der Habitus- und Milieuformen bei Lernenden und Lehrenden. Theoretische und methodologische Überlegungen zum Verhältnis von Habitus und sozialem Raum. In W. Helsper, R.-T. Kramer \& S. Thiersch (Hrsg.), Schülerhabitus. Theoretische und empirische Analysen zum Bourdieuschen Theorem der kulturellen Passung (S. 56-81). Wiesbaden: Springer VS.

Bremm, N., Klein, E. D., \& Racherbäumer, K. (2016). Schulen in „schwieriger“ Lage?! Begriffe, Forschungsbefunde und Perspektiven. DDS - Die Deutsche Schule, 108(4), 323-339.

Bremm, N., Racherbäumer, K., \& van Ackeren, I. (2017). Bildungsgerechtigkeit als Ausgangspunkt und Ziel ungleichheitsreflexiver Schulentwicklung in sozial deprivierten Kontexten. In B. Lütje-Klose, S. Miller, S. Schwab \& B. Streese (Hrsg.), Inklusion: Profile für die Schul- und Unterrichtsentwicklung in Deutschland, Österreich und der Schweiz. Theoretische Grundlagen - Empirische Grundlagen - Praxisbeispiele (S. 57-67). Münster: Waxmann.

Ditton, H. (2013). Kontexteffekte und Bildungsungleichheit: Mechanismen und Erklärungsmuster. In R. Becker \& A. Schulze (Hrsg.), Bildungskontexte. Strukturelle Voraussetzungen und Ursachen ungleicher Bildungschancen (S. 173-206). Wiesbaden: Springer VS.

Fabel-Lamla, M., \& Klomfaß, S. (2014). Lehrkräfte mit Migrationshintergrund. Habitussensibilität als bildungspolitische Erwartung und professionelle Selbstkonzepte. In T. Sander (Hrsg.), Habitussensibilität. Eine nеue Anforderung an professionelles Handeln (S. 209-228). Wiesbaden: Springer VS.

Fölker, L., \& Hertel, T. (2015). Differenz und Defizit. Rekonstruktionen zu pädagogischen Orientierungen und Praktiken der Klientelkonstruktion an zwei segregierten Großstadtschulen. In L. Fölker, T. Hertel \& N. Pfaff (Hrsg.), Brennpunkt(-)Schule. Zum Verhältnis von Schule, Bildung und urbaner Segregation (S. 105-122). Opladen: Barbara Budrich.

Fölker, L., Hertel, T., \& Pfaff, N. (2015). Schule ,im Brennpunkt‘ - Einleitung. In L. Fölker, T. Hertel \& N. Pfaff (Hrsg.), Brennpunkt(-)Schule. Zum Verhältnis von Schule, Bildung und urbaner Segregation (S. 9-26). Opladen: Barbara Budrich.

Grundmann, M., Groh-Samberg, O., Bittlingmayer, U., \& Bauer, U. (2003). Milieuspezifische Bildungsstrategien in Familie und Gleichaltrigengruppe. Zeitschrift für Erziehungswissenschaft (ZfE), 2003(1), 24-45.

Grundmann, M., Bittlingmayer, U.H., Dravenau, D., \& Groh-Samberg, O. (2007). Bildung als Privileg und Fluch - zum Zusammenhang zwischen lebensweltlichen und institutionalisierten Bildungsprozessen. In R. Becker \& W. Lauterbach (Hrsg.), Bildung als Privileg. Erklärungen und Befunde zu den Ursachen der Bildungsungleichheit (S. 47-74). Wiesbaden: Springer VS.

Häussermann, H. (2008). Wohnen und Quartier. Ursachen sozialräumlicher Segregation. In E.-U. Huster, J. Boeckh \& H. Mogge-Grotjahn (Hrsg.), Handbuch Armut und Soziale Ausgrenzung (S. 335-349). Wiesbaden: Springer VS.

Helsper, W. (2018). Lehrerhabitus. Lehrer zwischen Herkunft, Milieu und Profession. In A. Paseka, M. Keller-Schneider \& A. Combe (Hrsg.), Ungewissheit als Herausforderung für pädagogisches Handeln (S. 105-140). Wiesbaden: Springer VS.

Helsper, W., Kramer, R.-T., Hummrich, M., \& Busse, S. (2009). Jugend zwischen Familie und Schule. Eine Studie zu pädagogischen Generationsbeziehungen. Wiesbaden: Springer VS.

Hummrich, M. (2015). Schule und Sozialraum. Erziehungswissenschaftliche Perspektiven. In A. ElMafaalani, S. Kurtenbach \& K. P. Strohmeier (Hrsg.), Auf die Adresse kommt es an ... Segregierte Stadtteile als Problem- und Möglichkeitsräume begreifen (S. 168-187). Weinheim: Beltz Juventa.

Klein, E. D. (2017). Bedingungen und Formen erfolgreicher Schulentwicklung in Schulen in sozial deprivierter Lage. Eine Expertise im Auftrag der Wübben Stiftung. SHIP Working Paper Reihe, Bd. 01. Essen: Universität Duisburg-Essen.

Kramer, R.-T., \& Pallesen, H. (2018). Lehrerhandeln zwischen beruflichem und professionellem Habitus - Praxeologische Grundlegungen und heuristische Schärfungen. In T. Leonhard, J. Košinár \& C. Reintjes (Hrsg.), Praktiken und Orientierungen in der Lehrerbildung. Potentiale und Grenzen der Professionalisierung (S. 41-52). Bad Heilbrunn: Klinkhardt.

Kramer, R.-T., Helsper, W., Thiersch, S., \& Ziems, C. (2009). Selektion und Schulkarriere. Kindliche Orientierungsrahmen beim Übergang in die Sekundarstufe I. Wiesbaden: Springer VS.

Lange-Vester, A., \& Teiwes-Kügler, C. (2014). Habitussensibilität im schulischen Alltag als Beitrag zur Integration ungleicher sozialer Gruppen. In T. Sander (Hrsg.), Habitussensibilität. Eine neue Anforderung an professionelles Handeln (S. 177-208). Wiesbaden: Springer VS.

Mannheim, K. (1964). Wissenssoziologie. Neuwied: Luchterhand. 
Racherbäumer, K. (2017). Rekonstruktionen zu Bedeutung und Funktionen der Lehrer-Schüler-Beziehung aus Sicht von Lehrerinnen und Lehrern an Schulen in sozial benachteiligter Lage. In V. Manitius \& P. Dobbelstein (Hrsg.), Schulentwicklungsarbeit in herausfordernden Lagen (S. 123-139). Münster: Waxmann.

Rotter, C. (2014). Zwischen Illusion und Schulalltag. Berufliche Fremd- und Selbstkonzepte von Lehrkräften mit Migrationshintergrund. Wiesbaden: Springer VS. 